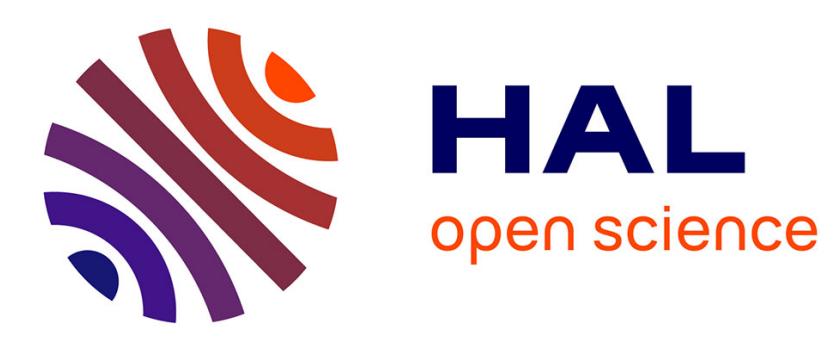

\title{
An importance measure to assess the value of a component inspection policy
}

\author{
William Fauriat, Enrico Zio
}

\section{To cite this version:}

William Fauriat, Enrico Zio. An importance measure to assess the value of a component inspection policy. 3rd International Conference on System Reliability and Safety, Nov 2018, Barcelone, Spain. 10.1109/ICSRS.2018.8688877 . hal-02056111

\section{HAL Id: hal-02056111 \\ https://hal-centralesupelec.archives-ouvertes.fr/hal-02056111}

Submitted on 4 Mar 2019

HAL is a multi-disciplinary open access archive for the deposit and dissemination of scientific research documents, whether they are published or not. The documents may come from teaching and research institutions in France or abroad, or from public or private research centers.
L'archive ouverte pluridisciplinaire HAL, est destinée au dépôt et à la diffusion de documents scientifiques de niveau recherche, publiés ou non, émanant des établissements d'enseignement et de recherche français ou étrangers, des laboratoires publics ou privés. 


\section{An importance measure to assess the value of a component inspection policy}

\author{
William Fauriat ${ }^{\mathrm{a}}$ \\ ${ }^{a}$ Chair System Science and the Energy Challenge, \\ Fondation Electricité de France (EDF) \\ CentraleSupélec, Université Paris-Saclay, France \\ william.fauriat@centralesupelec.fr
}

\author{
Enrico Zio ${ }^{\mathrm{a}, \mathrm{b}}$ \\ ${ }^{a}$ Chair System Science and the Energy Challenge, \\ Fondation Electricité de France (EDF) \\ CentraleSupélec, Université Paris-Saclay, France \\ ${ }^{b}$ Energy Department \\ Politecnico di Milano, Italy \\ enrico.zio@centralesupelec.fr \\ enrico.zio@polimi.it
}

\begin{abstract}
Importance measures have been used extensively to support risk-informed decision making. The purpose of such metrics is to help achieve safe and efficient design, maintenance and operation of assets in industrial sectors. In parallel, Value of Information (VoI), a concept at the junction of the fields of decision theory and Bayesian theory, has been used to define costefficient inspection policies, in particular in Structural Health Monitoring (SHM). This paper discusses the use of importance measures in a decision context that includes cost, maintenance and inspection, with the objective of defining infrastructure management policies. More specifically, it focuses on the use of VoI for the identification of components or groups of components that may be particularly 'information-relevant' in a given decision context.
\end{abstract}

Index Terms-Importance measures, Risk analysis, Probabilistic Risk Assessment (PRA), Infrastructure management, Decision theory, Value of Information, Inspection policy, Pre-posterior analysis

\section{INTRODUCTION}

Risk analysis and management methods provide the decision makers with tools for the design and operation of structures, systems and components. In order to guarantee the safety of critical systems, both the assessment of the potential risks associated with their operation and the choice of corresponding risk control measures must be carefully performed, with design and operation costs playing a role in the decision process.

Probabilistic Risk Assessment (PRA) is a widely used methodology that provides a quantitative description of the risk level associated to different undesirable scenarios. A PRA model is generally built from statistical knowledge available on the system. From the model, importance measures (IM) can be computed to characterize the role that the different components play for the risk of the system [1], [2].

Depending on the decision context, different importance measures can be used to produce different rankings of components, with respect to their contribution to the overall risk index at the system level, and identify safety-significant or risk-significant components [1]. Importance measures serve then as decision aids in the search for solutions to meet prescribed safety and budget requirements, see e.g. [3], [4]
Traditionally, importance measures are designed to quantify changes in terms of a risk metric and, thus, cannot be readily employed to compare different decisions in terms of costefficiency. The necessary extension of importance measures to a particular decision context has been considered by many authors, see e.g. [3]-[5] for examples and discussions. They can, then, be used to assess the interest of component reliability improvements or to evaluate different maintenance [6]-[8] or inspection policies.

For comparing the cost-efficiency of different inspection policies, the concept of Value of Information (VoI) is receiving increasing attention, especially in Structural Health Monitoring (SHM). Early works include [9], [10] and subsequent research activity has been developing since, see e.g. [11]-[13]. Essentially, VoI is a utility-based metric defined as the difference between the expected cost associated to the optimal decisions (e.g. maintenance) taken with and without the collection of additional information, through inspection or sensing, on the given structure. In association with adequate models for degradation and maintenance and inspection actions (e.g using Markov Decision Process (MDP)), an integrated approach for the elaboration of cost-efficient infrastructure management policies is proposed in [14].

The objective of this paper is to discuss Value of Information (VoI) concepts in the framework of Importance Measures (IM) for identifying components whose inspection or monitoring yields significant value in view of achieving a cost-efficient operation of the system.

The rest of the paper is organized as follows. Section II describes the importance measures traditionally considered and discusses the extension of their use into a decision context. Section III introduces the computation of VoI and proposes its application as a raking index for information-significance. Section IV proposes an example to illustrate the calculation and the practical use of IM and VoI for the cost-efficient operation of a system. Section V offers a look at the variation of inspection importance with the evolution of the decision context. Lastly, perspectives and conclusions for the use of a VoI-based metric for component inspection and/or monitoring are given in section VI. 


\section{IMPORTANCE MEASURES AND INFRASTRUCTURE MANAGEMENT CONTEXT}

\section{A. Definition and use of importance measures}

IMs quantify the effect of changes in the characteristics (here reliability is considered) of components or groups of components, on a risk index at the system level. Let $R_{s}$ be the risk index (here a reliability function) for a system with structure function $\Phi$. Let $\left(R_{i}=P\left(x_{i}=1\right)\right)_{i=1 \ldots p}$ be a set of probability values associated to the basic events $\left(x_{i}\right)_{i=1 \ldots p}$ representing the state $\left(x_{i}=0\right.$ if failed and $x_{i}=1$ if functioning) of the components. The system risk index is given by (1).

$$
R_{s}=\Phi\left(R_{1}, \ldots, R_{p}\right)
$$

For illustration purposes, a few common importance measures are defined in Table I, where $R_{0}$ is the value of the risk at the system level when the components' characteristics are at their nominal value $R_{i}^{0}=P\left(x_{i}=1\right)$. See e.g. [1] for details.

\begin{tabular}{lc}
\hline Measure & Definition \\
\hline Birnbaum importance & $R_{s}\left(R_{i}=1\right)-R_{s}\left(R_{i}=0\right)$ \\
Criticality importance & $\left(R_{s}\left(R_{i}=1\right)-R_{s}\left(R_{i}=0\right)\right) \times R_{i}^{0} / R_{0}$ \\
Risk Achievement Worth & $R_{s}\left(R_{i}=1\right) / R_{0}$ \\
Risk Reduction Worth & $R_{0} / R_{s}\left(R_{i}=0\right)$ \\
\hline
\end{tabular}

TABLE I

IMPORTANCE MEASURES

These measures describe the effect of large changes in the reliability of components from 0 to 1 . They may be used to illustrate the consequence of a component failure on the overall risk at the system level, thus giving an idea about the risk-significance of such component. As far as maintenance is concerned, they may be used to evaluate the effect of taking out a component for repair, or assess the worthiness of keeping a component highly reliable by specific maintenance measures. The use of importance measures as decision aids to identify, rank components or propose improvements [1], [2], is especially relevant when the system is large and his structure function (and associated PRA model) is complex, possibly including scores or tens hundreds of components.

Limitations usually cited when discussing the more traditional importance measures include:

- Their inability to describe small rather than large changes in the components' reliabilities or to deal with parametric descriptions which the latter could be based on.

- The fact that many of them cannot be used to handle groups of components but only components independently.

- The realization that they are based on 'point estimates' of the components' reliability and that the results that they give should be interpreted while considering the epistemic uncertainty associated to such estimates.

Developments have been proposed to overcome those limitations. As it is not the main purpose of this paper, the interested reader is refereed to existing literature. In [15], a so-called Differential Importance Measure (DIM) is proposed. The fact that it is an additive measure allows to consider groups of components and small changes or parametric descriptions for the components' reliability. More refined measures for the joint importance of two components are discussed in [16]. Extensive discussions about uncertainty importance and the effect of epistemic uncertainty are offered in [17], [18]. The use of decision theory is mentioned in [19] as a potential solution to measure and analyze uncertainty importance. A discussion about the connection between importance measures and a specific decision context is proposed hereafter.

\section{B. Extension of importance measures to a decision context}

In most cases, importance measures quantify changes in terms of a risk metric. Based on this information, the action that is subsequently taken has to consider the decision context. If a component ranks particularly high in terms of its influence on system reliability, a rational manager may want to augment the reliability of such component or modify the system's structure function, e.g. using redundancy. While such actions will likely increase the overall reliability of the system, they also induce a cost. Thus, the optimal solution is necessarily a tradeoff between the gain in reliability and the cost of realizing the improvements.

Let us define $L$ as the total cost (or loss, assuming that it is linearly related to monetary cost) associated to the operation of the system. Let us consider that this analysis is carried out in a design stage and that $R_{s}$ represents the probability that the system will operate properly during its operating life. Let us suppose that the cost of failure of the system may be estimated, through means outside of the scope of the present manuscript, and that its value is $c_{f}$. For example, if $N$ systems have been commercialized and the manufacturer must pay penalty fees (or warranty costs) for failed systems, then he may expect to pay $c_{\text {expected }}=N \times\left(1-R_{s}\right) \times c_{f}$. In that context and if one decides not to modify the system structure's function, looking for an optimal solution amounts to solving (2):

$$
\min _{\left\{R_{i}\right\} \in[0,1]^{p}} L\left(R_{1}, \ldots, R_{p}, c_{f}, c_{1}\left(R_{1}\right), \ldots, c_{p}\left(R_{p}\right)\right)
$$

where the cost function $L$ is defined in (3):

$$
L=c_{f} \times\left(1-R_{s}\left(R_{1}, \ldots, R_{p}\right)\right)+c_{1} R_{1}+\ldots+c_{p} R_{p}
$$

and $c_{i}:[0,1] \rightarrow \mathbb{R}^{+}$are monotonically increasing functions linking the reliability of component $i$ to the cost of producing it.

Extensions of traditional importance measures to a particular decision context (for example here, specifying the values $c_{f}$ and functions $c_{i}$ ) have been proposed, see e.g. [6]-[8]. In any case, the search for an optimal solution imposes that a decision context is specified (see [4] as an example).

The definition of a decision context and the interpretation of cost, reliability and risk values are neither simple problems nor is it the purpose of this paper to be exhaustive about modeling solutions to address such issues. 
From this point on, the value of these metrics do not characterize only the structure of a system or its constituents but also the cost of alternative actions and their consequences. They may be used to rapidly identify or rank the components based on the potential gain related to their improvement (or maintenance). A rather simple cost-based measure, quantifying the effect of changes on the cost function rather than the reliability function, is expressed in (4):

$$
\operatorname{LIM}=\frac{\partial L}{\partial R_{i}}
$$

which in the context defined in (3) gives (5):

$$
\operatorname{LIM}_{i}\left(R_{i}^{0}\right)=-c_{f} \frac{\partial R_{s}\left(R_{0}\right)}{\partial R_{i}}+\frac{\partial c_{i}\left(R_{i}^{0}\right)}{\partial R_{i}}
$$

Before moving to the next section, let us note that here, the reliability of the system $R_{s}$ is a scalar value. Nonetheless, the same reasoning may be applied to a time-varying function $R_{s}(t)$, if it is applied on different time instances (e.g. when considering life extension, see [10] for an example). The issue may become more difficult to address if multiple reparations can be counted, if downtime is costly and so on. In that case, one has to resort to more elaborate models, e.g using Markov Decision Process, see [14], [20]. The extension of importance measures to a time-varying context is theoretically possible but introducing parameters for the cost of one or different maintenance policies can prove much more complex. The reader may be interested in [3], [21] for measures taking into account the influence of time, outside of a decision context.

\section{VALUE OF INFORMATION AND INSPECTION IMPORTANCE}

\section{A. Value of Information}

For a structure or an industrial system, maintenance activities are meant to increase safety and expected life. In condition-based and predictive maintenance, decisions on when and what actions to perform are based upon the knowledge available on the aging state and behavior of the system and its components, derived from periodic inspection or continuous monitoring. By considering the total cost of the system's operation, including inspection and maintenance costs, one can choose among different policies. Value of Information (VoI) is a utility-based metric that may be used for that purpose.

As described in [10], "the value of a piece of information depends on its ability to guide our decision". VoI is a tool rooted both in Bayesian updating and decision theory that provides a rational approach to attach a value to a piece of information. Formally, VoI is the difference in expected cost (or loss) between the outcome of the best decisions that may be taken with and without the collection of additional information.

Let $L(s, a)$ be the loss function defined in relation with the system being in state $s$ and the manager choosing to apply action $a$ on the system (e.g. repairing). The VoI may be calculated according to (6):

$$
\mathrm{VoI}=L^{*}(\emptyset)-E_{O}\left[L^{*}(o)\right]
$$

where $L^{*}(\emptyset)$ represents the minimal expected loss (i.e. associated to the optimal decision) when no additional information is available and $L^{*}(o)$ represents the minimal expected loss when the information from observation $o$ is available, thus 'modifying' the prior knowledge on the aleatory uncertainty associated to the system state. As one does not know beforehand what the observation is going to be, $L^{*}(o)$ has to be averaged over all possible observations in set $O$. Giving the expression of the minimal expected loss and making the dependance on aleatory uncertainty explicit yields (7):

$$
\begin{aligned}
\mathrm{VoI}=\min _{a \in A} \int & L(s, a) p(s) d s \\
& -\int\left(\min _{a \in A} \int L(s, a) p(s \mid o) d s\right) p(o) d o
\end{aligned}
$$

where $A$ is the set of all possible actions, $p(s)$ is the prior distribution of the system states and $p(s \mid o)$ is the posterior distribution given an observation $o$.

Values for the observations are generally derived from an observation model. The latter accounts for the precision $p(o \mid s)$ of the inspection process (e.g. that of a sensor). The prior knowledge $p(s)$ on the system is generally used along with Bayes' equation $p(s \mid o)=p(o \mid s) p(s) / p(o)$ in order to sample from the observation distribution, see e.g. [10] for details. Hence, this approach is often called pre-posterior analysis.

The estimated VoI has to be compared to the cost of the acquisition of such information. Roughly speaking, the inspection is worth realizing if its cost does not exceed the additional value it can generate, namely VoI. This metric provides a rational approach to compare inspection devices or procedures that provide different pieces of information on the system state and with different levels of precision.

\section{B. Use of VoI for inspection importance assessment}

As expressed in section II-B, any extension of importance measures to a decision context implies the specification of such context. This is also the case for VoI as, according to (7), the computed VoI is necessarily dependent on:

- The decision context through the different possible actions and their outcomes $L(s, a)$

- The prior knowledge on the aleatory uncertainty associated to the system $p(s)$

- The precision of the inspection process $p(o \mid s)$

When the inspection consists in assessing the state of one or a group of components in the system, VoI can be seen as an importance measure, which we will call here: inspection importance measure (IIM). It serves as a decision aid allowing to rank different components based on the worthiness of obtaining information on any of them. While traditional importance measures quantify the effect on the risk metric at the system level, IIM quantifies the effect on the minimal expected 
cost (which can be lowered) when additional information on one or a group of components is available.

As the objective is to provide a metric for the comparison of different components, here, it will be assumed that one is able to obtain perfect information on the state of the components (failed or functioning). This allows us to leave aside considerations about inspection cost, generally related to the precision of the inspection procedure. In this case, the computed measure is the Value of Perfect Information (VoPI). The assumption of perfect information yields a value (IIM) that represents an upper bound on the potential gain in expected cost. One should note that in practice VoI will be lower than VoPI and that the inspection will have a nonnegligible cost. Yet, the higher the value of the IIM for a given component, the more valuable it is for the manager to collect information on it, in a given decision contest (i.e. when knowing the cost of potential actions such as maintenance).

\section{IMPORTANCE MEASURES ILLUSTRATION}

In this section, a simple system made of five components and displayed on Figure 1, is used as a means to illustrate the discussion of section II-B and the inspection importance measure (IIM) proposed in section III-B.

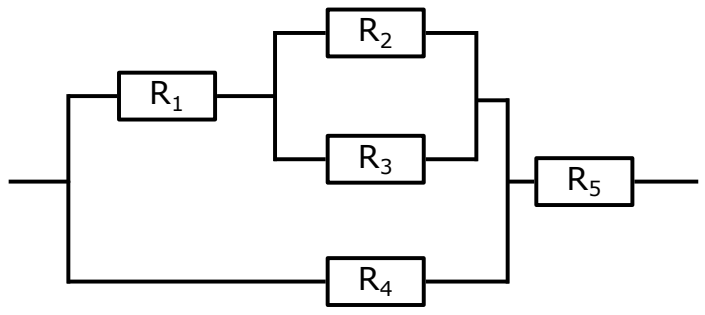

Fig. 1. Simple system with 5 components.

Components can be in either one of the two binary states, functioning or failed. The expression of the reliability function is given in (8), using the system structure function $\Phi$ :

$$
\begin{aligned}
R_{s}= & R_{4} R_{5}+R_{1} R_{2} R_{5}+R_{1} R_{3} R_{5} \\
& -R_{1} R_{2} R_{3} R_{5}-R_{1} R_{2} R_{4} R_{5}-R_{1} R_{3} R_{4} R_{5} \\
& +R_{1} R_{2} R_{3} R_{4} R_{5}
\end{aligned}
$$

where $\left(R_{i}\right)_{i=1 \ldots 5}$ constitute the prior knowledge on uncertainty associated to the system. Here, these reliability values describe the probability that the component is operating properly on a given time interval $[0, T]$ defining the life of the system.

\section{A. Importance measures without considering inspection}

Birnbaum importance measures are calculated for the different components and displayed on Figure 2. Epistemic uncertainty is introduced in the calculation by varying components reliabilities around their nominal values. The point is to illustrate that the ranking that one obtains using importance measures and a PRA model may be sensitive to the initial knowledge about the system. The nominal values for the components' reliability are given in Table II.

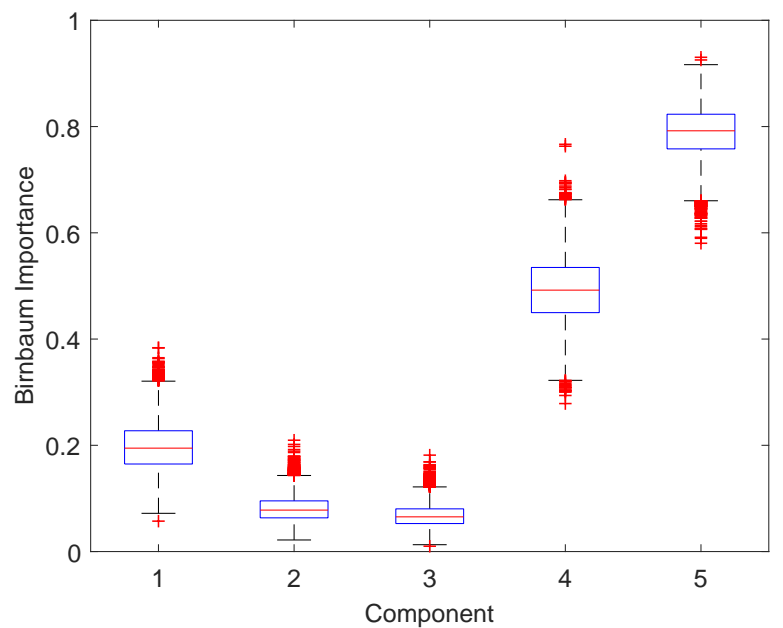

Fig. 2. Boxplot of Birnbaum Importance, with random variation of $\left(R_{i}\right)_{i=1 \ldots 5}$ around their nominal value (illustrating epistemic uncertainty).

Let us define $c_{f}$ as the cost induced by a failure of the system. Let $c_{R_{i}}$ be the cost associated to the maintenance action (e.g. repairing) applied on component $i$. Simply said, if cost $c_{R_{i}}$ is paid, component $i$ will not fail in $[0, T]$, i.e. $x_{i}=1$ and $R_{s}$ becomes $R_{s}^{i+}=R_{s}\left(x_{i}=1\right)$. The values for parameters $c_{f}$ and $c_{R_{i}}$ are given in Table II. In that context, the loss function is defined by (9):

$$
\begin{array}{r}
L(s, a)=c_{f} \times\left(1-\Phi\left(\max \left(x_{1}, a_{1}\right), \ldots,\right.\right. \\
\left.\left.+\max \left(x_{5}, a_{5}\right)\right)\right) \\
+\sum_{i=1 \ldots 5} a_{i} c_{R_{i}}
\end{array}
$$

where $s=\left(x_{i}\right)_{i=1 \ldots 5}$ characterizes the state of the system and $a=\left(a_{i}\right)_{i=1 \ldots 5}$ is the action vector with $a_{i}=1$ if a repair action is applied for component $i$ and $a_{i}=0$ otherwise. With five components there are $2^{5}=32$ potential actions to consider. $\Phi$ is the system's structure function.

\begin{tabular}{lr}
\hline Parameter & Value \\
\hline$R_{1}, R_{2}, R_{3}, R_{4}, R_{5}$ & $0.6,0.5,0.5,0.7,0.9$ \\
$c_{f}$ & 500 \\
$c_{R_{1}}, c_{R_{2}}, c_{R_{3}}, c_{R_{4}}, c_{R_{5}}$ & $20,10,15,40,60$ \\
\hline
\end{tabular}

TABLE II

NOMINAL VALUES FOR THE COMPUTATION OF IMPORTANCE MEASURES.

On Figure 3, the average outcome $\left(E_{S}[L(s, a)]\right)$ of different actions is illustrated.

The first five values on the upper part of Figure 3 $\left(\left(\mathrm{LIM}_{i}\right)_{i=1, \ldots, 5}=(20.5,10.25,5.25,34.25,-18.25)\right)$ can be seen as a cost-based importance measure for the five different components. The measure represents the average gain when repairing such components (or assuring perfect maintenance on $[0, t]$ ) without any specific 'inspection' information, given 


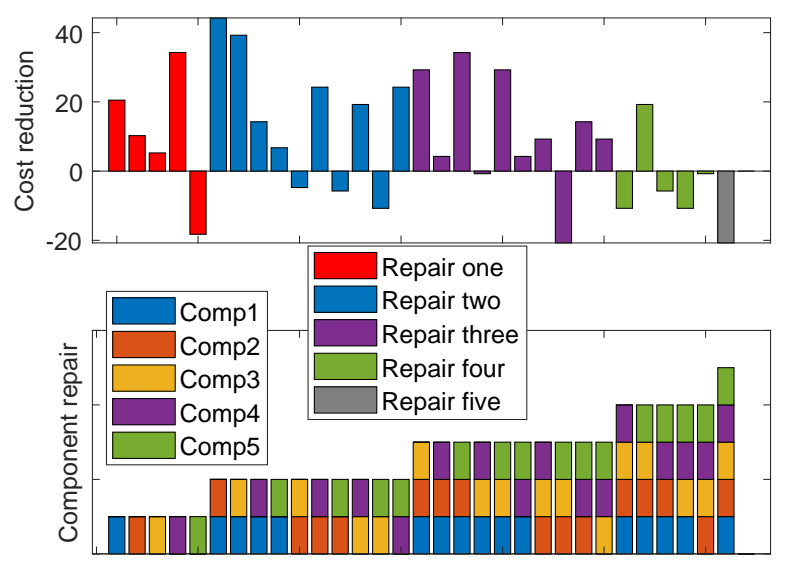

Fig. 3. Potential gain in expected cost (upper part) when repairs are made (with respect to the situation with no repairs). Lower part of the figure describes which components are repaired.

the decision context and associated costs. These values may be calculated according to (10):

$$
\operatorname{LIM}_{i}=c_{f} \times\left[R_{s}^{i+}-R_{0}\right]-c_{R_{i}}
$$

where $R_{s}^{i+}=R_{s}\left(x_{i}=1\right)$ is the reliability of the system when component $i$ is maintained perfectly functional at $\operatorname{cost} c_{R_{i}}$.

It is interesting to notice that the decision context can alter the relative importance of components obtained when based only on system structure and components reliability. As an example, component 5 Birnbaum importance value is large on Figure 2, while its cost-based importance $\mathrm{LIM}_{5}=-18.25$ points to a negative outcome of maintaining it. This can be explained by a high 'base' reliability $R_{5}^{0}$ and a significant maintenance cost $c_{R_{5}}$. Lastly, on Figure 3 , one can also consider the effect of pairs of components, thus assessing joint importance.

\section{B. Inspection importance measure (IIM)}

The decision context defined in section IV-A is introduced in order to propose a VoI-based measure, labeled as IIM, that can be used to aid in the identification of components whose inspection holds particular value. In practice, it may be difficult to assign a value to system failure cost or maintenance costs. The latter may be associated to a specific preventive maintenance plan on the entire time interval $[0, t]$. Nonetheless, it is interesting to use the VoI concept to address the following question: if I could have 'clairvoyance' on the true state (here the outcome on the interval $[0, t]$ ) of the considered system (i.e. a given individual in the population $p(s)$ ), what should action I select: maintain or not, one or several of its components.

Let us assume that one is able to acquire perfect information on the system at no cost. Here the objective is not actually to judge an inspection process but rather to rank components based on the worthiness of acquiring information on them. If component $i$ is inspected, the related inspection importance measure (IIM) can be calculated according to (11):

$$
\begin{aligned}
& \mathrm{IIM}_{i}=\min _{a}\left(c f \times\left(1-R_{s}^{a}\right)+\sum a_{i} c_{R_{i}}\right) \\
&-\min _{a}\left(c f \times\left(1-R_{s}^{a, i+}\right)+\sum a_{i} c_{R_{i}}\right) \times R_{i}^{0} \\
&-\min _{a}\left(c f \times\left(1-R_{s}^{a, i-}\right)+\sum a_{i} c_{R_{i}}\right) \times\left(1-R_{i}^{0}\right)
\end{aligned}
$$

where $R_{s}^{a}=\Phi\left(\max \left(R_{1}, a_{1}\right), \ldots, \max \left(R_{5}, a_{5}\right)\right)$ is the value of the reliability function modified by action $a, R_{s}^{a, i+}=$ $R_{s}^{a}\left(R_{i}=1\right)$ and $R_{s}^{a, i-}=R_{s}^{a}\left(R_{i}=0\right)$ are the values of the reliability function when the inspection guarantees that component $i$ is either functioning or failed and action $a$ is applied. In this context of perfect inspection $R_{i}^{0}$ and $\left(1-R_{i}^{0}\right)$ are used as the probabilities of observing component $i$ in functioning or failed state, based on the prior knowledge on the system $\left(R_{i}\right)_{i=1, \ldots, 5}$.

The expression for joint inspection of components is notgiven but it can be deduced easily from (7) as (11) was. Results of the calculation of IIMs are given on Figure 4. The first five values of the upper part of Figure $4\left(\left(\mathrm{IIM}_{i}\right)_{i=1, \ldots, 5}=\right.$ $(12,5,5,21,44))$ are inspection importance measures for the five different components.

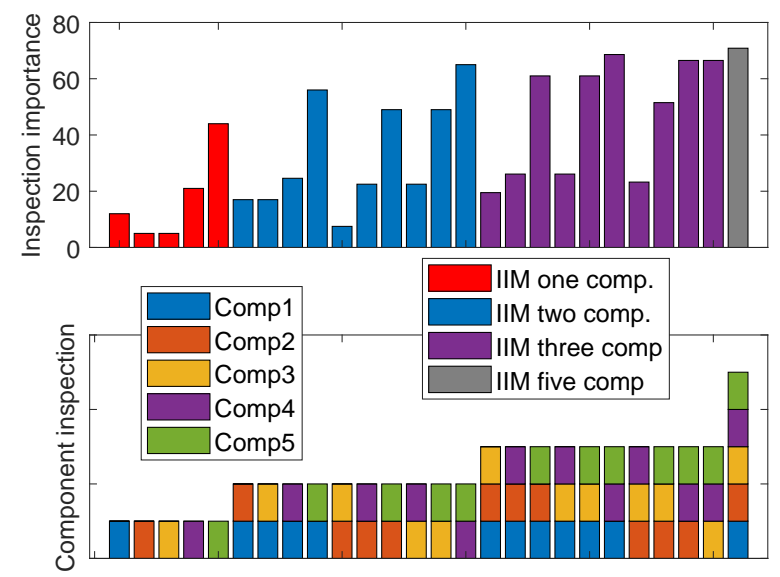

Fig. 4. Inspection importance measures (upper part) based on the inspection of one or several components (Lower part of the figure describes which components are inspected).

With the values considered in Table II, the minimal expected cost is $L^{*}(\emptyset)=80$ and the VoPI for a complete inspection of all components is VoPI $=70.85$. This mean that, through inspections, the minimal expected cost of operating the system can be brought down to $L^{*}=9.15$. Simply said, this corresponds to the outcome where repair actions are only taken when specifically needed. In practice, $L^{*}$ is a lower bound that cannot be reached exactly, as one has to consider inspection costs and limited precision or partial nature of the inspections.

As can be expected, inspecting several components at the same times yields higher value. It can also be noted that 
combinations of inspections that correspond to a minimal path set: here $(4,5),(1,2,5)$ or $(1,3,5)$ are particularly valuable. Taken alone, component 5 ranks as the highest IIM value. This may be explained by the fact that it is a critical component which contributes immediately to the failure of the system, and that its repair cost is large and repairing only the failed individuals (or rather individual which will fail on $[0, t]$ ), thanks to inspections, does bring the overall cost down. The influence of the decision context, especially costs values, is further illustrated in the following section.

\section{INFLUENCE OF CHANGE IN THE DECISION FRAMEWORK ON INSPECTION IMPORTANCE}

In order to get an idea on the effect of the parameters that may influence IIM, the latter are calculated with different values for:

- Prior knowledge (i.e. here $\left.\left(R_{i}\right)_{i=1, \ldots, 5}\right)$

- Repair / maintenance costs

- Precision of the inspection

The influence of the repair costs on IIMs is illustrated on Figures 5 through 7. $L^{*}$ is defined on these Figures as the expected cost when no inspection if carried out and it can be diminished when information is collected. VoPI results correspond to the perfect inspection of all five components, thus defining the maximal potential gain in this decision context.

A couple of remarks can be made. First, it is seen that when the repair cost of component 5 increases (in fact in relation with a fixed failure cost of $c_{f}=500$ ), at one point it becomes too costly to repair this component without specific information on its state. When component 5 repair cost is close to failure cost, inspection information is worthless, as it becomes non-profitable to repair component 5 regardless of its actual state. Conversely when component 5 repair cost is low, one might as well repair it, regardless of the knowledge one has on the true state of component 5. For both those extremes there is little or no value in inspection. Second, it can be noticed on the upper part of Figure 6 that the ranking between the five components evolves when the repair costs vary. Third, on Figure 5, variations in component 5 repair cost only influence $\mathrm{IIM}_{5}$ and not the IIMs of the other components. But this is not always the case, as can be seen on Figure 7 for the effect of component 4 repair cost.

The prior knowledge on the system uncertainty, here the reliability of the components, also has an effect on the computed IIMs. On Figures 8 and 9 the following remarks can be made. First, as the reliability of component 5 increases the minimal expected cost $L^{*}$ decreases. The system becomes more cost-efficient thanks to the higher performances of its components (obviously cost of component 5 has to be taken into account also). Second, if one knows that the reliability of component 5 is low, it will generally be necessary to repair it, and inspecting will not produce much cost saving. Conversely, if it is expected to be very reliable, most inspections will give the same answer, making inspection less valuable. Yet one has

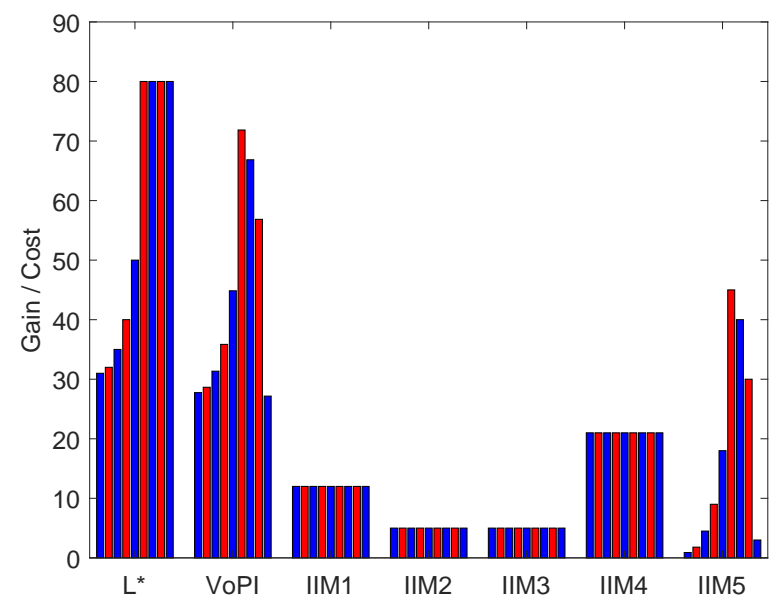

Fig. 5. Influence of repair cost (ranging from 1 to 500) of component 5 on IIM and $L^{*}$.
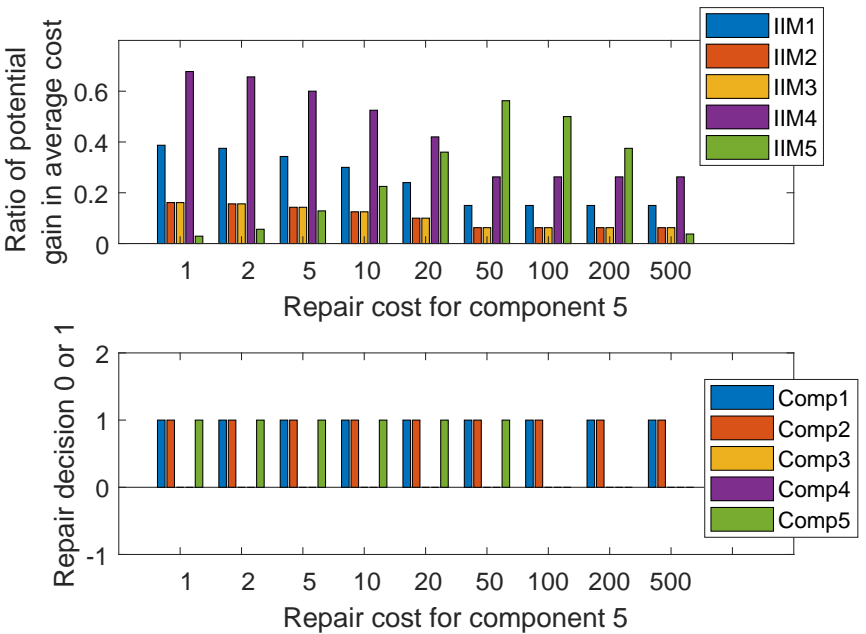

Fig. 6. Ratio of potential gain in expected cost (with respect to $L^{*}$ ) with additional information, as a function of the repair cost (ranging from 1 to 500 ) of component 5 (top). Best average decision $a^{*}$ (vector with 0s or 1s) for each value of component 5 repair cost (bottom).

to remain careful with this analysis, as it may no longer be valid if repair cost is simultaneously high.

It is also interesting to consider the influence of the precision of the inspection process. As previously mentioned, IIMs are upper bounds on the potential gain achievable through inspection. On Figure 10, one sees that the VoI increases with the precision. It is also good to note that the ranking of the different components, as far as information-significance is concerned, does not seem to depend too much on precision. Thus IIMs, corresponding to perfect information, are valuable metrics to make rankings between the different components of a system, in a given decision context.

Finally, let us point out that in the framework of this paper and for the calculation of IIMs, here, the repair or maintenance actions are considered perfect. Indeed, if $\operatorname{cost} c_{R_{i}}$ is paid, 


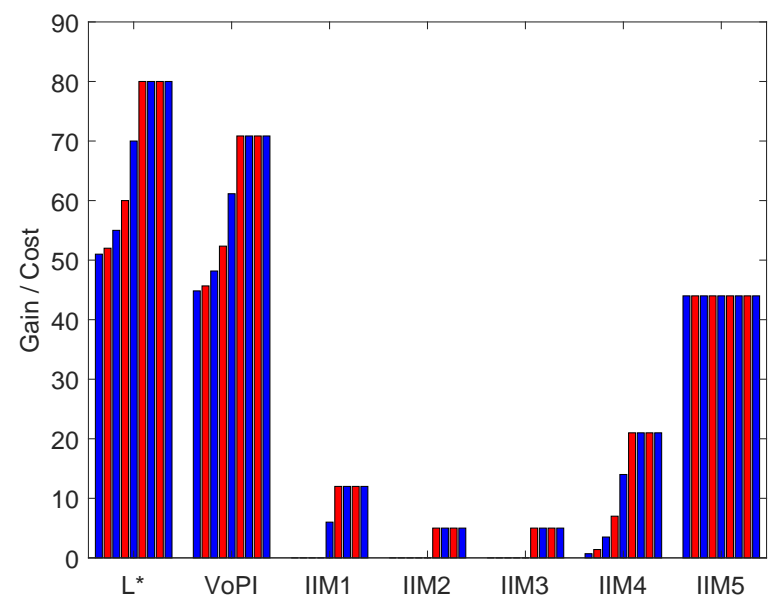

Fig. 7. Influence of repair cost (ranging from 1 to 500) of component 4 on IIM and $L^{*}$.

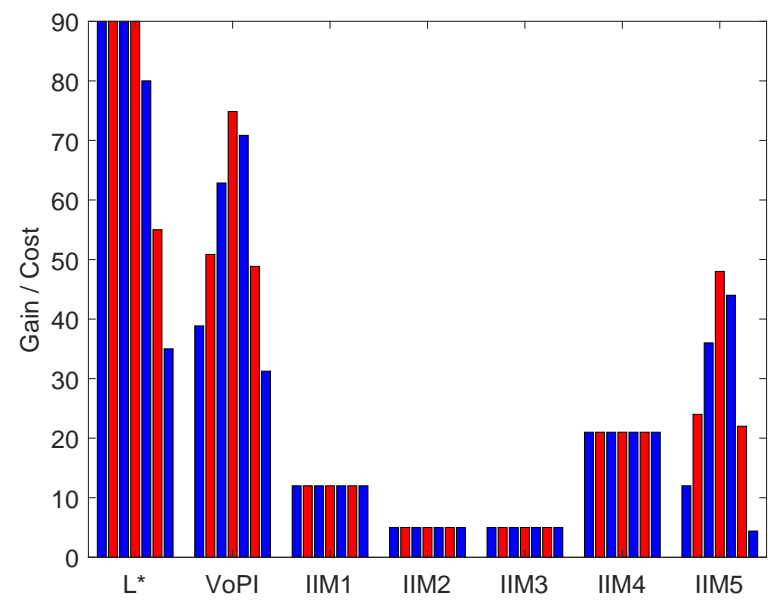

Fig. 8. Influence of reliability (ranging from 0.2 to 0.99 ) of component 5 on IIM and $L^{*}$.

then, reliability of component $i$ is fixed to its maximum value $R_{i} \leftarrow 1$ on time interval $[0, T]$. This must not be considered as an absolute restriction and (11) can still be applied if the so-called 'maintenance action' consists in assuring a given reliability of component $i$, which needs not be perfect $R_{i}<1$.

\section{CONCLUSION}

Importance measures have been used extensively to rank or identify components with significant impact on the risk index at the system level. Such measures can be used as decision aids to propose improvements on the system structure, on the reliability of components or on maintenance policies. The decision taken with this information will likely be a tradeoff between assuring a high level of reliability for the system and the cost to realize improvement actions. Thus, extensions of importance measures to the decision context have to include a precise specification of the latter. This
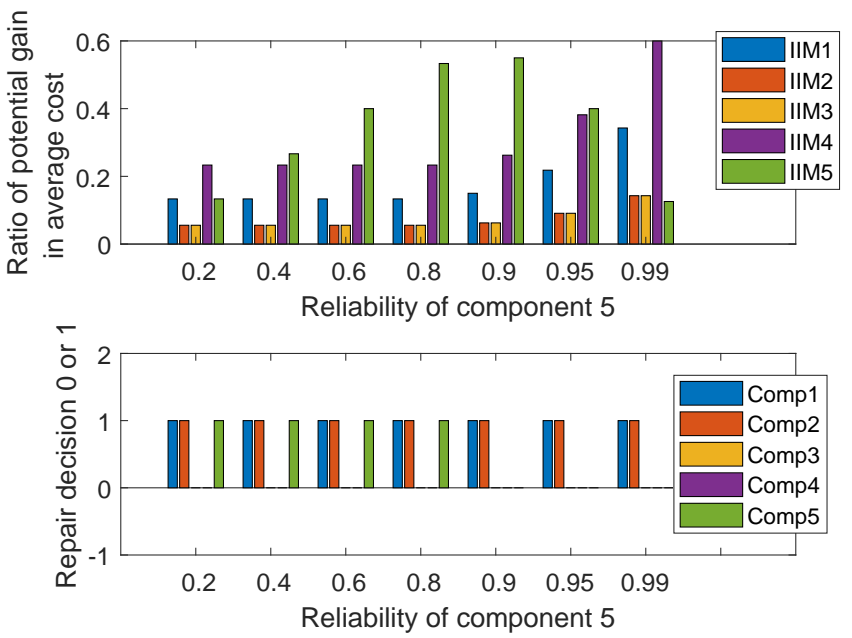

Fig. 9. Ratio of potential gain in expected cost (with respect to $L^{*}$ ) with additional information, as a function of the reliability (ranging from 0.2 to 0.99 ) of component 5 (top). Best average decision $a^{*}$ (vector with 0 s or $1 \mathrm{~s}$ ) for each value of component 5 reliability (bottom).

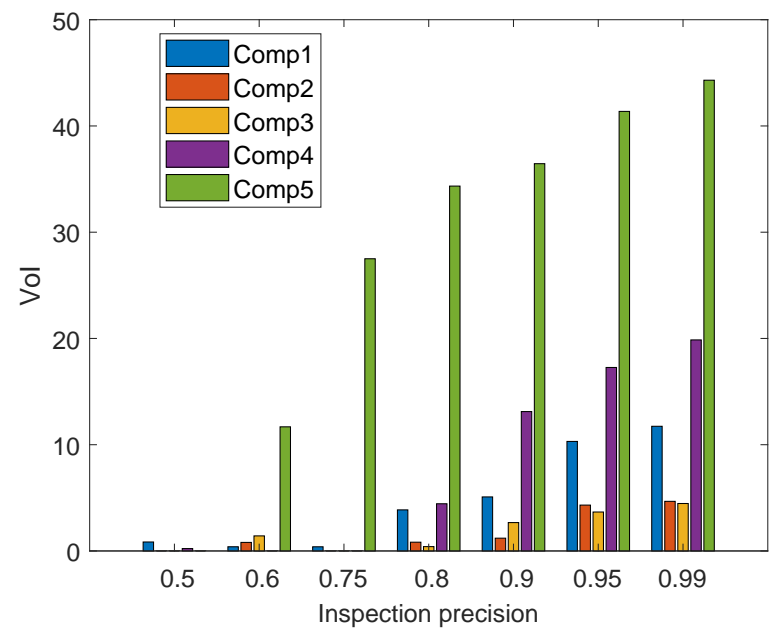

Fig. 10. VoI for different values of the inspection precision (ranging from 0.5 : non-informative test, to 0.99 ).

includes the specification of the cost of system failure as well as the specification of the alternative actions, their costs and their outcomes. Let us point out that this specification phase can constitute a significant increase in modeling work on top of the construction of the PRA model.

As far as operation decisions are concerned, the inclusion of specific information about a given individual of interest, i.e. inspection, within a population for which one possess prior knowledge, is an efficient way to reach a lower expected cost. When it comes to setting up a monitoring or inspection policy, it is important to weight inspection costs against the value of the information that may be acquired from inspection. It is, then, relevant to use a VoI-based metric to identify components whose inspection is particularly valuable for the cost-efficient operation of the system. A measure, based on 
the assumption of perfect information, has been proposed here. It has been coined 'inspection importance measure' (IIM) and computations in the case of a simple example have demonstrated its ability to rank components according to their 'information-significance'.

This approach is particularly attractive in a context where condition-monitoring (CM) data is increasingly available and may be processed into valuable and individual-specific (i.e. among a population of systems) information. IIM are an interesting tool to rapidly identify which components of a complex system should receive particular attention, in a given decision context, when additional information can be collected for such components. In this paper, the specification of the decision context has not been extensively developed and a straightforward formulation has been sought for simplicity and clarity's sake. Obviously this constitutes a difficult issue in practice but, nonetheless, the underlying concept of a VoIbased metric can always be applied. A study on the effect of the parameters that may influence the computed IIM has been carried out, in order to provide elements to consider regarding that last issue.

Future work on this topic include the application of information-related measures in situations where inspection information originates from the processing of CM data. In this case, information extracted from CM is likely imperfect and one has to account for the precision of the diagnostics or prognostics model, which should be assessed beforehand, when computing any VoI or VoI-based metric. Additionally, the handling of time-varying problems, involving complex policies (combinatorial complexity of the action space might be very large) on a given time horizon, has only been mentioned here. In this latter framework, the use of more complex tools, e.g. MDP models, or the formulation of a decision problem adapted to the time-varying context, may be necessary.

\section{ACKNOWLEDGMENT}

The authors acknowledge the support of the Chair System Science and the Energy Challenge, Fondation Electricite de France (EDF)

\section{REFERENCES}

[1] M. C. Cheok, G. W. Parry, and R. R. Sherry, "Use of importance measures in risk-informed regulatory applications," Reliability Engineering \& System Safety, vol. 60, no. 3, pp. 213-226, 1998.

[2] M. Van der Borst and H. Schoonakker, "An overview of PSA importance measures," Reliability Engineering \& System Safety, vol. 72, no. 3, pp. 241-245, 2001.

[3] X. Zhu and W. Kuo, "Importance measures in reliability and mathematical programming," Annals of Operations Research, vol. 212, no. 1, pp. 241-267, 2014.

[4] E. Zio and L. Podofillini, "Importance measures and genetic algorithms for designing a risk-informed optimally balanced system," Reliability Engineering \& System Safety, vol. 92, no. 10, pp. 1435-1447, 2007.

[5] W. Kuo and X. Zhu, "Some recent advances on importance measures in reliability," IEEE Transactions on Reliability, vol. 61, no. 2, pp. 344 360,2012

[6] S. Wu and F. P. A. Coolen, "A cost-based importance measure for system components: an extension of the birnbaum importance," European Journal of Operational Research, vol. 225, no. 1, pp. 189-195, 2013.
[7] S. Wu, Y. Chen, Q. Wu, and Z. Wang, "Linking component importance to optimisation of preventive maintenance policy," Reliability Engineering \& System Safety, vol. 146, pp. 26-32, 2016.

[8] H. Dui, S. Si, and R. C. M. Yam, "A cost-based integrated importance measure of system components for preventive maintenance," Reliability Engineering \& System Safety, vol. 168, pp. 98-104, 2017.

[9] D. Straub and M. H. Faber, "Risk based inspection planning for structural systems," Structural safety, vol. 27, no. 4, pp. 335-355, 2005.

[10] M. Pozzi and A. Der Kiureghian, "Assessing the value of information for long-term structural health monitoring," in Health monitoring of structural and biological systems 2011, vol. 7984, p. 79842W, International Society for Optics and Photonics, 2011.

[11] D. Zonta, B. Glisic, and S. Adriaenssens, "Value of information: impact of monitoring on decision-making," Structural Control and Health Monitoring, vol. 21, no. 7, pp. 1043-1056, 2014.

[12] S. Thöns, "On the value of monitoring information for the structural integrity and risk management," Computer-Aided Civil and Infrastructure Engineering, vol. 33, no. 1, pp. 79-94, 2018.

[13] D. Straub, E. Chatzi, E. Bismut, W. Courage, M. Döhler, M. H. Faber, J. Köhler, G. Lombaert, P. Omenzetter, M. Pozzi, et al., "Value of information: a roadmap to quantifying the benefit of structural health monitoring," in ICOSSAR-12th International Conference on Structural Safety \& Reliability, 2017.

[14] M. Memarzadeh and M. Pozzi, "Value of information in sequential decision making: component inspection, permanent monitoring and systemlevel scheduling," Reliability Engineering \& System Safety, vol. 154, pp. 137-151, 2016.

[15] E. Borgonovo and G. E. Apostolakis, "A new importance measure for risk-informed decision making," Reliability Engineering \& System Safety, vol. 72, no. 2, pp. 193-212, 2001.

[16] E. Zio and L. Podofillini, "Accounting for components interactions in the differential importance measure," Reliability Engineering \& System Safety, vol. 91, no. 10-11, pp. 1163-1174, 2006.

[17] T. Aven and K. Pörn, "Expressing and interpreting the results of quantitative risk analyses. review and discussion," Reliability Engineering \& System Safety, vol. 61, no. 1-2, pp. 3-10, 1998.

[18] E. Borgonovo, "Measuring uncertainty importance: investigation and comparison of alternative approaches," Risk analysis, vol. 26, no. 5, pp. 1349-1361, 2006.

[19] K. Pörn, "A decision-oriented measure of uncertainty importance for use in PSA," Reliability Engineering \& System Safety, vol. 56, no. 1, pp. 17-27, 1997.

[20] K. G. Papakonstantinou and M. Shinozuka, "Planning structural inspection and maintenance policies via dynamic programming and markov processes. part i: theory," Reliability Engineering \& System Safety, vol. 130, pp. 202-213, 2014.

[21] H. Peng, D. W. Coit, and Q. Feng, "Component reliability criticality or importance measures for systems with degrading components," IEEE Transactions on Reliability, vol. 61, no. 1, pp. 4-12, 2012. 